\title{
允许修改推理规则的开放逻辑 *
}

\author{
应明生 \\ (江西师范大学数学系, 南昌 330027)
}

\section{关镜词开放逪暂 推理规则 面构}

最近, 李未 ${ }^{[1]}$ 为了刻划知识的增长、更新以及假说的进化建立了一个开放的逻辑理论, 特别是得到了认知进程的收玫性定理这样一个深刻的结果. 其后, 他在文献 [2] 中又将其 作为逻辑框架应用于知识基的维护. 在知识库中, 除了一些事实 (相当于句子) 作为知识 之外, 还有其特有的一些推理规则, 而且这些推理规则有时也会遇到反驳, 需要加以修改和 维护. 本文试图在开放逻辑中引入推理规则的反驳与修改. 值得注意的是, 其它一些关于 知识基维护的理论, 如 Alchourrón, Gärdenfors 与 Makinson ${ }^{\left[{ }^{3}{ }^{3}\right.}$ 都没有触及这个问题.

定义 1 设 $\varphi \in W f f, \varphi$ 的自由变元都在 $x_{1}, \cdots, x_{n}$ 中. 如果对任意 $a_{1}, \cdots a_{n} \in|M|(M$ 的论域), 总有 $M \vDash \varphi\left[a_{1}, \cdots, a_{n}\right]$, 则记 $M \vDash \varphi$.

(1) 设 $r$ 是 $n$ 元 (Hilbert 型) 推理规则 ${ }^{[4]}$, 即 $r$ 是映射 $D(r) \subseteq W f f^{n} \rightarrow W f f$. 称模型 $M$ 满足 $r$, 记 $M \vDash r$, 如果对任意 $\left(\varphi_{1}, \cdots, \varphi_{n}\right) \in D(r)$, 当 $M \vDash \varphi_{i}, i=1, \cdots, n$ 时, $M \vDash r\left(\varphi_{1}, \cdots\right.$, $\left.\varphi_{n}\right)$. 若 $\Gamma$ 是一集推理规则, 则 $M \vDash \Gamma$ 表示对任意 $r \in \Gamma, M \vDash r$;

(2) 一集推理规则 $\Gamma$ 称为和谐的当且仅当存在模型 $M \vDash \Gamma$;

(3) 设 $\Gamma$ 是一集推理规则, $r$ 是一个推理规则, 称 $r$ 可由 $\Gamma$ 语义导出, 记 $\Gamma \vDash r$, 若对任意 模型 $M$, 当 $M \vDash \Gamma$ 时, 也有 $M \vDash r$.

命题 1 记 $T(r)=\left\{\bigwedge_{i=1}^{n} c\left(\varphi_{i}\right) \rightarrow c\left(r\left(\varphi_{1}, \cdots, \varphi_{n}\right)\right) \mid\left(\varphi_{1}, \cdots, \varphi_{n}\right) \in D(r)\right\}$, 这里 $c(\varphi)$ 表示 $\varphi$ 的闭包, 即当 $\varphi$ 的自由变元都在 $x_{1}, \cdots, x_{n}$ 中时, $c(\varphi)=\forall x_{1} \cdots \forall x_{n} \varphi$; 又记 $T(\Gamma)=$ $\bigcup_{r \in \Gamma} T(r)$.

(1) $M \vDash \Gamma$ 当且仅当 $M \vDash T(\Gamma)$;

(2) $\Gamma$ 是和谐的当且仅当 $T(\Gamma)$ 和谐;

(3) $\Gamma \vDash r$ 当且仅当 $T(\Gamma) \vDash T(r)$.

系 1 (1)若 $\Gamma$ 的每个有限子集和谐,则 $\Gamma$ 亦和谐;

(2) 设 $r$ 是有限的, 即 $D(r)$ 有限. 若 $\Gamma \vDash r$, 则存在 $\Gamma$ 的有限子集 $\Delta \vDash r$.

定义 2 推理规则 $r$ 称为闭的, 若 $D(r), R(r)$ 中都只包含句子, 和谐的非空闭推理规则 集称为假说. 闭推理规则 $r$ 称为假说 $\Gamma$ 的新假设, 若 $\Gamma \cup\{r\}$ 和谐且 $\Gamma \not \neq r$.

定义 3 设 $\Gamma$ 是假说, $r$ 是闭规则. 若 $r$ 是可满足的, 即存在模型 $M \vDash r$, 又 $\Gamma \cup\{r\}$ 不和

1994-12-17 收稿, 1995-10-16 收修改稿

*国家“863”高技术研究发展计划资助项目 
谐,则称使 $\Delta U\{r\}$ 和谐的 $\Gamma$ 的极大子集 $\Delta$ 为 $\Gamma$ 关于 $r$ 的可接受子集.

命题 2 每个使 $\Delta U\{r\}$ 和谐的 $\Gamma$ 的子集 $\Delta$ 都可以扩充为 $\Gamma$ 关于 $r$ 的可接受子集.

命题 3 若 $\Delta$ 为 $\Gamma$ 关于 $r$ 的可接受子集,则存在使 $\Theta$ 和谐的 $T(\Gamma)$ 的极大子集 $\Theta$ 满足 $\Delta=\{s \in \Gamma \mid T(s) \subseteq \Theta\}$.

注 有反例说明命题 3 之逆不成立. 由此可见, 推理规则的修改问题并不能完全化归为 公式的情形加以处理.

定义 4 设 $\Gamma$ 是假说, $r$ 是闭规则.

(1) 若 $\Gamma \vDash r$, 则 $\Gamma \cup\{r\}$ 称为 $\Gamma$ 的一个正常扩充;

（2）若 $r$ 是 $\Gamma$ 的新假设,则 $\Gamma \cup\{r\}$ 称为 $\Gamma$ 的一个 $N$-重构;

(3) 若 $r$ 可满足, $\Gamma \cup\{r\}$ 不和谐且 $\Delta$ 是 $\Gamma$ 关于 $r$ 的可接受子集,则 $\Delta \cup\{r\}$ 称为 $\Gamma$ 的一 个 $R$-重构.

定义 5 设 $\alpha \in O_{n}$ (序数). 假说序列 $\left\{\Gamma_{\beta}\right\}_{\beta<\alpha}$ 称为 $\alpha$ 长的认知进程, 如果对任意 $\beta<\alpha$.

（1）当 $\beta=\gamma+1$ 时, $\Gamma_{\beta}$ 为 $\Gamma_{\gamma}$ 的一个正常扩充、 $N$-重构或 $R$-重构;

(2) 当 $\beta \in O_{n_{\Pi}}$ (极限序数) 时,

$$
\underline{\lim }\left\{\Gamma_{\gamma}\right\}_{\gamma<\beta} \subseteq \Gamma_{\beta} \subseteq \varlimsup\left\{\Gamma_{\gamma}\right\}_{\gamma<\beta},
$$

这里

$$
\begin{aligned}
& \left.\underline{\lim }\left\{\Gamma_{\gamma}\right\}_{\gamma<\beta}=|r| \text { 存在 } \gamma<\beta \text { 使对任意 } \delta \geqslant \gamma, r \in \Gamma_{\delta}\right\} \text {, } \\
& \widetilde{\lim }\left\{\Gamma_{\gamma}\right\}_{\gamma<\beta}=\left\{r \mid \text { 对任意 } \gamma<\beta \text {, 存在 } \delta \geqslant \gamma \text {, 使 } r \in \Gamma_{\delta}\right\} \text {. }
\end{aligned}
$$

注 (1) 当 $\alpha>\omega$ 时,认知进程本身中就包含有极限过程, 这可以看作认知产生了(多次) 飞跃.

（2）将 $\Gamma_{\gamma}$ 作为 $\Gamma_{\gamma+1}$ 的认识基础. 当 $\Gamma_{\gamma} \vDash r$ 时, 虽然 $r$ 可由 $\Gamma_{\gamma}$ 推出, 但这种推导可能十 分困难 (比如数学中一些定理的获得) ; 在认识过程中, 人们往往将 $r$ 加入 $\Gamma_{\gamma}$ 中也作为新的认 知基础. 因此, 在定义 5 的情形(1)中, 允许 $\Gamma_{\gamma+1}$ 为 $\Gamma_{\gamma}$ 的正常扩充.

定理 1 给定模型 $M$ 及假说 $\Gamma$, 总存在长度 $\leqslant 2^{\|L\|}$ 的认知进程 $\left\{\Gamma_{\beta}\right\}$ 使 $\Gamma_{0}=\Gamma$ 且 $\lim \left\{\Gamma_{\beta}\right\}=$ $\mathscr{R}_{M}$, 这里 $\mathscr{R}_{M}$ 是 $M$ 所满足的所有闭推理规则之集.

证 将 $\mathscr{R}_{M}$ 排为序列 $\left\{r_{\beta}\right\}_{\beta<\alpha}\left(\alpha \leqslant_{2}\|L\|\right)$, 总可设 $\alpha \in O_{n_{\mathbb{I}}}$. 令 $\Gamma_{0}=\Gamma$, 并设 $\Gamma_{\beta}$ 已经定 义且满足 $\left\{r_{\theta}\right\}_{\theta<\beta} \subseteq \Gamma_{\beta}$, 则 $\Gamma_{\beta+1}$ 定义如下:

情形 $1 \quad \Gamma_{\beta} \vDash r_{\beta}$ 或 $\Gamma_{\beta} U\left\{r_{\beta}\right\}$ 和谐且 $\Gamma_{\beta} \mid \neq r_{\beta}$. 令 $\Gamma_{\beta+1}=\Gamma_{\beta} \cup\left\{r_{\beta}\right\}$.

情形 $2 \Gamma_{\beta} \bigcup\left\{r_{\beta}\right\}$ 不和谐. 由于 $M \vDash r_{\beta}, r_{\beta}$ 可满足. 因为 $\left(r_{\theta}\right\}_{\theta<\beta} \cup\left\{r_{\beta}\right\}$ 和谐, 由命题 2, 存在 $\Gamma_{\beta}$ 关于 $r_{\beta}$ 的可接受子集 $\Delta_{\beta} \supseteq\left\{r_{\theta}\right\}_{\theta<\beta}$, 令 $\Gamma_{\beta+1}=\Delta_{\beta} \cup\left\{r_{\beta}\right\}$.

当 $\beta \in O_{n_{\text {II }}}$ 时, 令 $\Gamma_{\beta}=\underline{\lim }\left\{\Gamma_{\gamma}\right\}_{\gamma<\beta}$. 显然, $\left\{\Gamma_{\beta}\right\}_{\beta<\alpha}$ 是认知进程且 $\underline{\lim }\left\{\Gamma_{\beta}\right\} \supseteq \mathscr{R}_{M}$. 以下往 证 $\overline{\lim }\left\{\Gamma_{\beta}\right\} \subseteq \mathscr{R}_{M}$. 因为 $\overline{\lim }\left\{\Gamma_{\beta}\right\}$ 的元素都是闭推理规则, 只需证若闭推理规则 $r \notin \mathscr{R}_{M}$, 则 $r \notin$ $\overline{\lim }\left\{\Gamma_{\beta}\right\}$. 这可证明如下: 若闭规则 $r \notin \mathscr{R}_{M}$, 则 $\mathscr{R}_{M} \cup\{r\}$ 不和谐. 事实上, 若反设 $\mathscr{R}_{M} \cup\{r\}$ 和 谐, 即存在 $M^{\prime} \vDash \mathscr{R}_{M} \cup\{r\}$, 则 $M^{\prime} \vDash \mathscr{R}_{M}$. 将每个句子看作一个零元的闭推理规则, 则对任意句 子 $A$, 当 $M \vDash A$ 时, 总有 $M^{\prime} \vDash A$. 如此, 当 $M^{\prime} \vDash A$ 时, $M^{\prime} \not \neq 7 A$, 从而 $M \not \neq 7 A$, 即 $M \vDash A$. 因此, $M$ 与 $M^{\prime}$ 初等等价. 再由 $M^{\prime} \vDash r$ 得 $M \vDash r$ (注意 $r$ 是闭推理规则, 并参见命题 1(1)), 即 $r \in \mathscr{R}_{M}$, 矛盾. 现在, 由 $\mathscr{R}_{M} \cup\{r\}$ 不和谐及命题 1 的系 1(1) 得知, 存在 $r_{\beta_{1}}, \cdots, r_{\beta_{n}}$, 使 $\beta_{i}<\alpha$ 
$(i=1, \cdots, n)$ 且 $\left\{r, r_{\beta_{1}}^{*}, \cdots, r_{\beta_{n}}\right\}$ 不和谐. 令 $\beta_{0}=\max \left\{\beta_{1}, \cdots, \beta_{n}\right\}$, 由于 $\alpha \in O_{n_{\text {II }}}, \beta_{0}<\alpha$, 有 $\beta_{0}+$ $2<\alpha$. 因为 $r_{\beta_{1}}, \cdots, r_{\beta_{n}} \in \Gamma_{\beta_{0}+1}$, 则 $r \notin \Delta_{\beta_{0}+1}$. 由 $\Gamma_{\beta}$ 的定义知, 当 $\delta \geqslant \beta_{0}+2$ 时, $r \notin \Gamma_{\delta}$, 从而 $r \notin \widetilde{\lim }\left\{\Gamma_{\beta}\right\}$.

为了进一步地研究开放逻辑中推理规则的反驳与修改,需要将模型论中的理论用闭推理 规则集代替而获得一些相应的结论,这实际上给模型论提出了一类新的问题. 另外, 本文只考 虑了 Hilbert 型推理规则, 还有必要讨论 Gentzen 型推理规则 ${ }^{[4]}$ 的反驳与修改.

\section{参考文献}

1 本 未.一个开放的逻辑系统. 中国科学, A 辑, 1992, (10):1 103 1114

2 Li W. A logical framework for knowledge base maintenance. J of Comput Sci \& Technol, 1995, 10(3): 193 205

3 Alchourrón C E, Gärdenfors R, Makinson D. On the logic of theory change: partial meet contraction and revision functions. J Symbolic Logic, 1985, 50(2): 510 530

4 Wojjcicki R. Theory of Logical Calculi : Basic Theory of Consequence Operations. Dordrecht: Kluwer Academic Publishers, 1988 